\title{
PbrRALF2-elicited reactive oxygen species signaling is mediated by the PbrCrRLK1L13- PbrMPK18 module in pear pollen tubes
}

Xiaobing Kou', Jiangmei Sun ${ }^{1}$, Peng Wang ${ }^{1}$, Danqi Wang ${ }^{1}$, Peng Cao ${ }^{1}$, Jing Lin², Youhong Chang ${ }^{2}$, Shaoling Zhang ${ }^{1}$ and Juyou $\mathrm{Wu}^{1,2 \times}$

\begin{abstract}
Rapid alkalinization factors (RALFs) are cysteine-rich peptides that play important roles in a variety of biological processes, such as cell elongation and immune signaling. Recent studies in Arabidopsis have shown that RALFs regulate pollen tube growth via plasma membrane receptor-like kinases (RLKs). However, the downstream signal transduction mechanisms of RLKs in pollen tubes are unknown. Here, we identified PbrRALF2, a pear (Pyrus bretschneideri) pollen RALF peptide that inhibits pollen tube growth. We found that PbrRALF2 interacts with a malectin-like domain-containing RLK, PbrCrRLK1L13. The relative affinity between PbrRALF2 and PbrCrRLK1L13 was at the submicromolar level, which is consistent with the values of ligand-receptor kinase pairs and the physiological concentration for PbrRALF2-mediated inhibition of pollen tube growth. After binding to its extracellular domain, PbrRALF2 activated the phosphorylation of PbrCrRLK1L13 in a dose-dependent manner. We further showed that the MAP kinase PbrMPK18 is a downstream target of PbrCrRLK1L13 that mediates PbrRALF2-elicited reactive oxygen species (ROS) production. The excessive accumulation of ROS inhibits pollen tube growth. We show that MPK acts as a mediator for CrRLK1L to stimulate ROS production, which might represent a general mechanism by which RALF and CrRLK1L function in signaling pathways.
\end{abstract}

\section{Introduction}

The pollen tube is a tubular structure used to transport sperm to the ovule for fertilization. This process requires crosstalk between pollen tubes and the pistil, which ensures that the pollen tube grows normally until reaching the ovule. The germination of a pollen grain and the subsequent rapid elongation of a pollen tube are regulated by multiple factors, such as extracellular peptides, hormones, and intracellular signals of calcium ions and reactive oxygen species. Among these factors, pollensecreted peptides, which are multiple signaling molecules,

\footnotetext{
Correspondence: Juyou Wu (juyouwu@njau.edu.cn)

${ }^{1}$ Center of Pear Engineering Technology Research, State Key Laboratory of

Crop Genetics and Germplasm Enhancement, College of Horticulture, Nanjing Agricultural University, 210095 Nanjing, China

2Jiangsu Key Laboratory for Horticultural Crop Genetic Improvement, 210014 Nanjing, China

These authors contributed equally: Xiaobing Kou, Jiangmei Sun, Peng Wang
}

play important roles in the development of pollen tubes, such as LAT52 $2^{1}$, phytosulfokines ${ }^{2}$, and rapid alkalinization factors (RALFs) ${ }^{3,4}$. RALFs are cysteine-rich peptides (CRPs) of $\sim 5 \mathrm{kDa}^{5}$. Treatment of pollen with extracellular RALF inhibits pollen tube elongation, which is mediated through membrane receptors ${ }^{4,6,7}$.

BUPS1 and BUPS2 were recently identified as RALF receptors ${ }^{4,6}$, which are expressed in mature pollen grains and tubes. BUPS1 and BUPS2 belong to the rose periwinkle (Catharanthus roseus) receptor protein kinase (CrRLK1L) family, which is conserved in plants and has 17 members in Arabidopsis. CrRLK1Ls have been proposed to act as 'sensor' proteins. For example, FERONIA (FER) is a receptor for RALF1 and RALF23 in Arabidopsis, and it is involved in signaling for root elongation ${ }^{8,9}$, immunity ${ }^{10}$, and fertilization in female tissue ${ }^{11,12}$. In addition, the FER orthologs ANXUR1 (ANX1) and

\section{(c) The Author(s) 2021}

(c) (i) Open Access This article is licensed under a Creative Commons Attribution 4.0 International License, which permits use, sharing, adaptation, distribution and reproduction cc) in any medium or format, as long as you give appropriate credit to the original author(s) and the source, provide a link to the Creative Commons license, and indicate if changes were made. The images or other third party material in this article are included in the article's Creative Commons license, unless indicated otherwise in a credit line to the material. If material is not included in the article's Creative Commons license and your intended use is not permitted by statutory regulation or exceeds the permitted use, you will need to obtain permission directly from the copyright holder. To view a copy of this license, visit http://creativecommons.org/licenses/by/4.0/. 
ANXUR1 (ANX2) are required to regulate pollen tube integrity ${ }^{13-18}$. Notably, the bups 1 and bups 1 bups 2 lossof-function mutants show precocious pollen tube rupture, similar to the phenotype of the anx1 anx2 double mutant ${ }^{6,14}$. Despite this progress in understanding CrRLK1L family functions, the downstream signal transduction mechanisms of these receptor-like kinases in pollen tubes are poorly understood.

Mitogen-activated protein kinases (MPKs) are among the downstream targets of RALF signaling ${ }^{5}$, but the MPKs involved in the RALF response and how they are activated remain unknown. Some MPKs are known to be involved in transducing RLK signaling in other processes ${ }^{19,20}$; for example, MPK3 and MPK6 are downstream targets of (salt intolerance 1) SIT1 in rice and mediate salt-induced ethylene signaling ${ }^{21}$. Furthermore, a YODA-MKK4/ MPK5-MPK3/MPK6 module regulates inflorescence architecture downstream of ERECTA-RLK ${ }^{22}$. ROS are also important messengers in RLK signaling ${ }^{23}$. ANX1 and ANX2 redundantly increase the activity of NADPH oxidases to generate ROS. ROS generation is indispensable for steady pollen tube elongation and helps to maintain the cell wall integrity of the pollen tube ${ }^{24}$. RALF17-based induction of ROS production is mediated by FER ${ }^{10}$. These results suggest that the suppression of pollen tube growth by RALF signaling could be determined by CrRLK1Lmediated ROS production.

In this study, we identified a pear pollen self-generated RALF peptide, PbrRALF2, and its receptor, PbrCrRLK1L13. We further found that after the direct interaction between ligand and receptor, PbrRALF2 promoted the phosphorylation of PbrCrRLK1L13, which subsequently recruited PbrMPK18 to produce ROS. The subsequent excessive accumulation of ROS inhibited pollen tube growth. The results indicate that a RALF-CrRLK1L-MPK-ROS pathway transduces extracellular signaling to the cytosol, thereby maintaining the moderate growth rate of the pollen tube in an autoregulatory manner.

\section{Results}

\section{PbrRALF2 inhibits pear pollen tube growth}

Using HMMER3 software with the RALF conserved domain PF05498 as a query, we identified 24 candidate $R A L F$ genes in the "Dangshansuli" pear genome and assigned them names based on the nomenclature and numbering conventions used for the RALF genes in Arabidopsis (Supplementary Table S1). Using reverse transcription PCR (RT-PCR), we found that 16 of the 24 PbrRALF genes were highly expressed in pollen (Supplementary Fig. S1A). We expressed and purified the 16 pollen-expressed PbrRALFs using an Escherichia coli system (Supplementary Fig. S1B). These purified recombinant proteins were used to treat pear pollen, and we observed that PbrRALF2, PbrRALF7, and PbrRALF11 significantly inhibited the growth of pollen tubes, with PbrRALF2 showing the largest effect (Fig. 1A). Based on these results, we selected PbrRALF2 for subsequent experiments.

PbrRALF2 is expressed at a low level in multiple tissues, including the roots, stems, leaves, fruits, petals, and pistils, and is highly expressed in pollen (Supplementary Fig. S2A). To further explore the inhibition of pollen tube growth by PbrRALF2, we tested a synthetic PbrRALF2 peptide along with the purified recombinant PbrRALF2 described above for dose-dependent effects. The concentrations of PbrRALF2 required for 50\% inhibition $\left(\mathrm{IC}_{50}\right)$ of pollen tube growth were $58 \pm 20 \mathrm{nM}$ and $92 \pm$ $12 \mathrm{nM}$ for synthetic and E. coli-expressed PbrRALF2, respectively (Fig. 1B).

We next used an antisense oligonucleotide (as-ODN) approach to knock down gene expression, which is widely used in pollen tube genetic analysis ${ }^{25-28}$ to downregulate PbrRALF2. Significant promotion of pollen tube length was observed in PbrRALF2-as-ODN, where PbrRALF2 was knocked down, but not in the presence of the corresponding sense oligonucleotides (Fig. 1C). These results suggest that PbrRALF2 could inhibit pollen tube growth.

\section{PbrCrRLK1L13 is a receptor for PbrRALF2}

To identify the components in PbrRALF2 signaling, we performed a yeast two-hybrid ( $\mathrm{Y} 2 \mathrm{H})$ screen using PbrRALF2 as bait and a pear pollen cDNA library as prey. One of the candidates was Pbr001839.1 (Supplementary Table S2), which encodes PbrCrRLK1L13 of the pear CrRLK1L family ${ }^{29}$. CrRLK1Ls are membrane proteins that consist of an extracellular domain (ECD), a transmembrane domain (TM), and an intracellular kinase domain (IKD) ${ }^{30}$. Consistent with PbrCrRLK1L13 being a membrane protein, when we expressed PbrCrRLK1L13 in Arabidopsis protoplasts as a fusion with green fluorescence protein, it localized to the plasma membrane (Supplementary Fig. S3).

Although $\operatorname{PbrCrRLK1L13}$ from pear, RUPO from rice (Oryza sativa), and BUPS1 (At4g39110) and BUPS2 (At2g21480) from Arabidopsis belong to the same phylogenetic clade ${ }^{29}$, RUPO, BUPS1, and BUPS2 have pollenspecific expression in rice ${ }^{16}$ and Arabidopsis ${ }^{6}$ while PbrCrRLK1L13 expression is observed in multiple tissues in pear (Supplementary Fig. S3A). To confirm the physical interaction between PbrRALF2 and PbrCrRLK1L13, we used two different approaches. A Y2H assay revealed that PbrRALF2 directly interacted with PbrCrRLK1L13 (Fig. 2A), and a deletion analysis delineated the interaction domain to the exJM of PbrCrRLK1L13 (Fig. 2B). No obvious interaction between PbrRALF2 and three other PbrCrRLK1L proteins, PbrCrRLK1L19 (homolog of THE1 in Arabidopsis), PbrCrRLK1L10 (homolog of HERK1 in Arabidopsis), and PbrCrRLK1L9 (homolog of HERK2 in Arabidopsis), was observed (Supplementary Fig. S4A). Moreover, both 


\section{A}

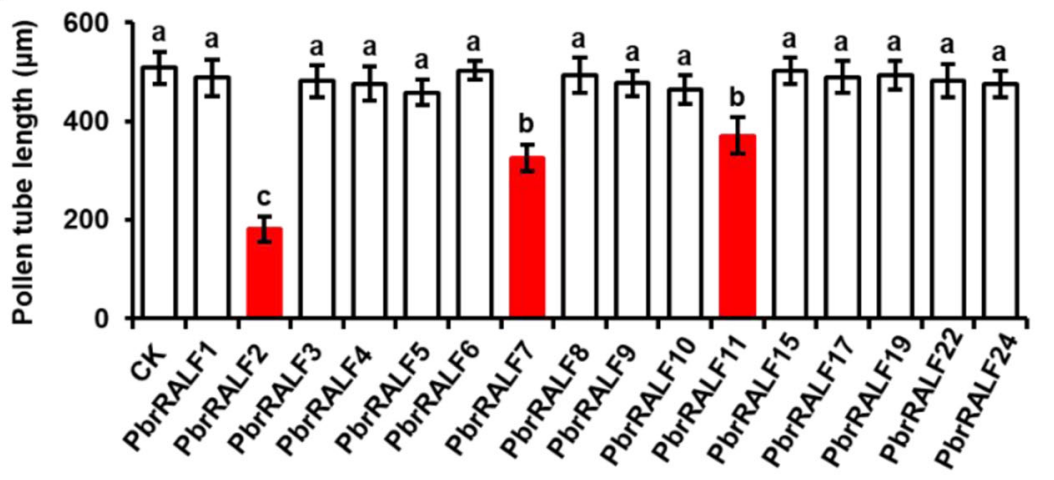

B
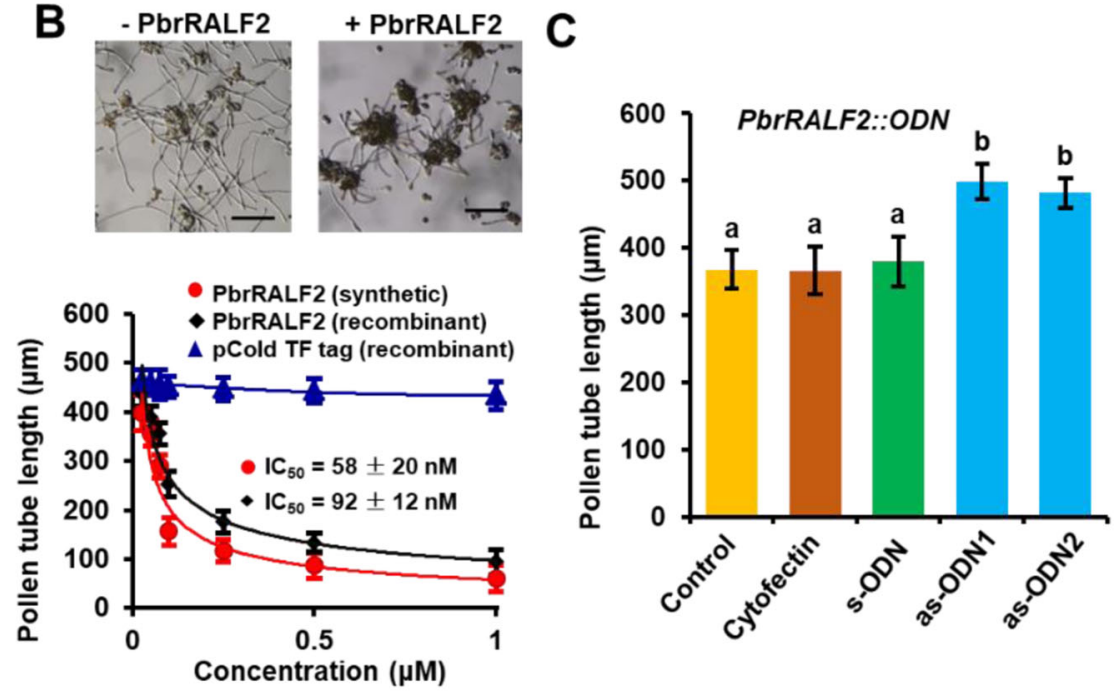

Fig. 1 PbrRALFs inhibit pollen tube growth. A Pollen tubes from pear were treated with $1 \mu \mathrm{M}$ recombinant PbrRALFs, and pollen tube growth was measured. Selected PbrRALFs were expressed in pollen by RT-PCR (Supplementary Fig. S1A). Different letters indicate significant differences, as determined by one-way ANOVA $(P<0.05, n=3)$. Three independent experiments were conducted. Data points are presented as the means \pm s.e.m. with those significantly different from the control (CK) shown in red. B Recombinant or synthetic PbrRALF2 at different concentrations $(0.025 \mu \mathrm{M}$, $0.05 \mu \mathrm{M}, 0.075 \mu \mathrm{M}, 0.1 \mu \mathrm{M}, 0.25 \mu \mathrm{M}, 0.5 \mu \mathrm{M}$, and $1 \mu \mathrm{M}$ ) was added to precultured pollen tubes. Images shown were acquired $10 \mathrm{~h}$ after treatment without or with purified recombinant PbrRALF2. Scale bars $=0.1 \mathrm{~mm}$. Data points are presented as the means \pm s.e.m. $\left(I_{50}=58 \pm 20 \mathrm{nM}\right.$ for synthetic PbrRALF2 and $92 \pm 12 \mathrm{nM}$ for recombinant PbrRALF2). C Knockdown (antisense oligodeoxynucleotide; as-ODN) of the expression of PbrRALF2 promoted pollen tube growth $(P<0.05, n=3)$, whereas s-ODN (sense ODN) and cytofection control had no function. UT indicates no treatment controls. Different letters indicate significant differences, as determined by one-way ANOVA $(P<0.05, n=3)$. Three independent experiments were conducted

PbrRALF7 and PbrRALF11, the other two PbrRALFs that showed an inhibitory effect on pollen tube growth (Fig. 1A), also interacted with PbrCrRLK1L13 in Y2H assays (Supplementary Fig. S4B). Furthermore, we tested six PbrRALFs that showed no effects on pollen tube growth and found no interaction with PbrCrRLK1L13 (Supplementary Fig. S4C). Thus, these results support the direct interaction between PbrRALF2 and PbrCrRLK1L13.

We used surface plasmon resonance (SPR) and isothermal titration calorimetry (ITC) to determine the affinity of PbrRALF2 and PbrCrRLK1L13 for each other. The purified recombinant PbrRALF2 bound to PbrCrRLK1L13 with dissociation constant $(K d)$ values of
$73 \pm 17$ and $512 \pm 21 \mathrm{nM}$, as revealed by SPR (Fig. 2C) and ITC (Fig. 2D), respectively. These values are consistent with those reported for other ligand-receptor kinase pairs ${ }^{10,31}$ and the $\mathrm{IC}_{50}$ for PbrRALF2-mediated inhibition of pollen tube growth (Fig. 1B). Thus, we conclude that PbrCrRLK1L13 could be a receptor for PbrRALF2.

\section{PbrRALF2 increases PbrCrRLK1L13 phosphorylation and ROS production}

We further examined whether PbrCrRLK1L13 kinase activity is affected by PbrRALF2. PbrRALF2 could induce PbrCrRLK1L13 phosphorylation, suggesting that the binding of PbrRALF2 to PbrCrRLK1L13 is physiologically 

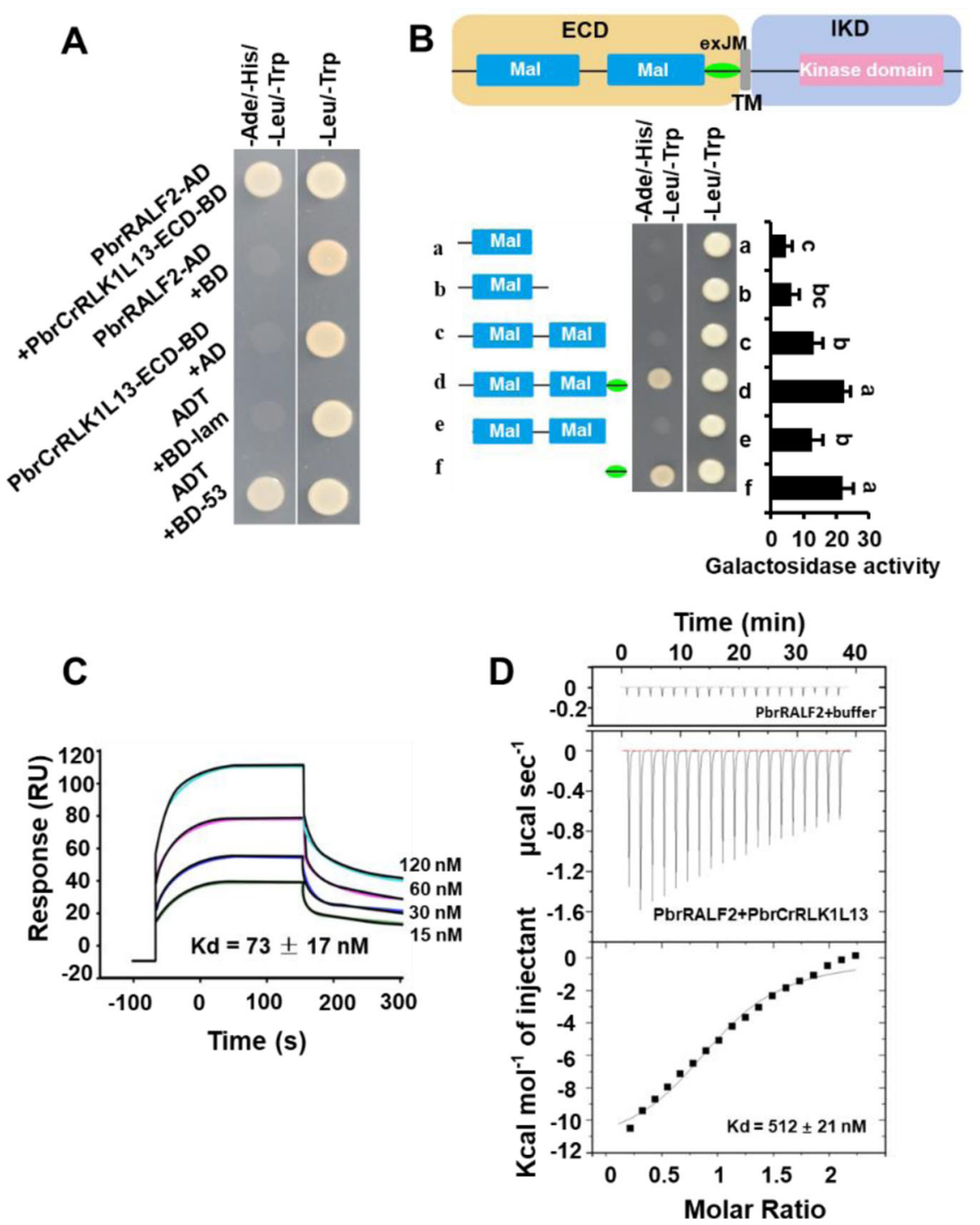

Fig. 2 Binding of PbrRALF2 to PbrCrRLK1L13. A PbrRALF2 interacts with the extracellular domain of PbrCrRLK1L13 in yeast. PbrRALF2 was fused to the pGADT7 vector, and the extracellular domain of PbrCrRLK1L13 was fused to the pGBKT7 vector. Growth in the medium that lacked Trp, Leu, His, and Ade indicated protein-protein interactions. B Extracellular juxtamembrane (exJM) region of PbrCrRLK1L13 is required for the PbrRALF2 interaction. The predicted structure of PbrCrRLK1L13, including the extracellular domain (ECD), exJM, transmembrane domain (TM), and intracellular kinase domain (IKD). Dissection of the exJM region of PbrCrRLK1L13 is required for the interaction with PbrRALF2 in a yeast two-hybrid assay. The interaction strength was quantified through $\beta$-galactosidase activity. Different letters indicate significant differences, as determined by one-way ANOVA $(P<0.05, n=3)$. C Binding of four concentrations of PbrRALF2 to PbrCrRLK1L13 in the surface plasmon resonance (SPR) assay. Different colored lines represent the titration of different concentrations of PbrRALF2 protein in the SPR assay. The light blue line indicates the $120 \mathrm{nM}$ PbrRALF2 assay, the pink line indicates the $60 \mathrm{nM}$ PbrRALF2 assay, the dark blue line indicates the $30 \mathrm{nM}$ PbrRALF2 assay, and the green line indicates the $15 \mathrm{nM}$ PbrRALF2 assay. The black lines represent the fitting line. The solid line is a fit with Michaelis-Menten kinetics, yielding an apparent dissociation constant of $\mathrm{Kd}=73 \pm 17 \mathrm{nM}$. D Quantitative binding analysis using PbrRALF2 and the extracellular domain of PbrCrRLK1L13 using isothermal titration calorimetry (ITC). A representative thermogram was obtained from $200 \mu$ M PbrRALF2 titrations into $20 \mu M$ PbrCrRLK1L13. Nonlinear regression of the PbrRALF2 vs. PbrCrRLK1L13 dosage yielded an apparent dissociation constant of Kd $=512 \pm 21 \mathrm{nM}$

functional (Fig. 3A, B). The level of PbrCrRLK1L13 phosphorylation increased in a PbrRALF2 dosedependent manner (Fig. 3C, D). However, the phosphorylation levels of PbrCrRLK1L13 were not affected by PbrRALF19, even when the concentration of PbrRALF19 was increased to $2 \mu \mathrm{g}$ (Fig. 3C, D).
We observed a significant promotion of pollen tube length in the presence of as-ODN targeted to the PbrCrRLK1L13 sequence but not in the presence of s-ODN (Fig. 4A). Moreover, the growth of the PbrCrRLK1L13-knockdown pollen tube was less sensitive to PbrRALF2 (Fig. 4B). Similar to RALF17, which induces 


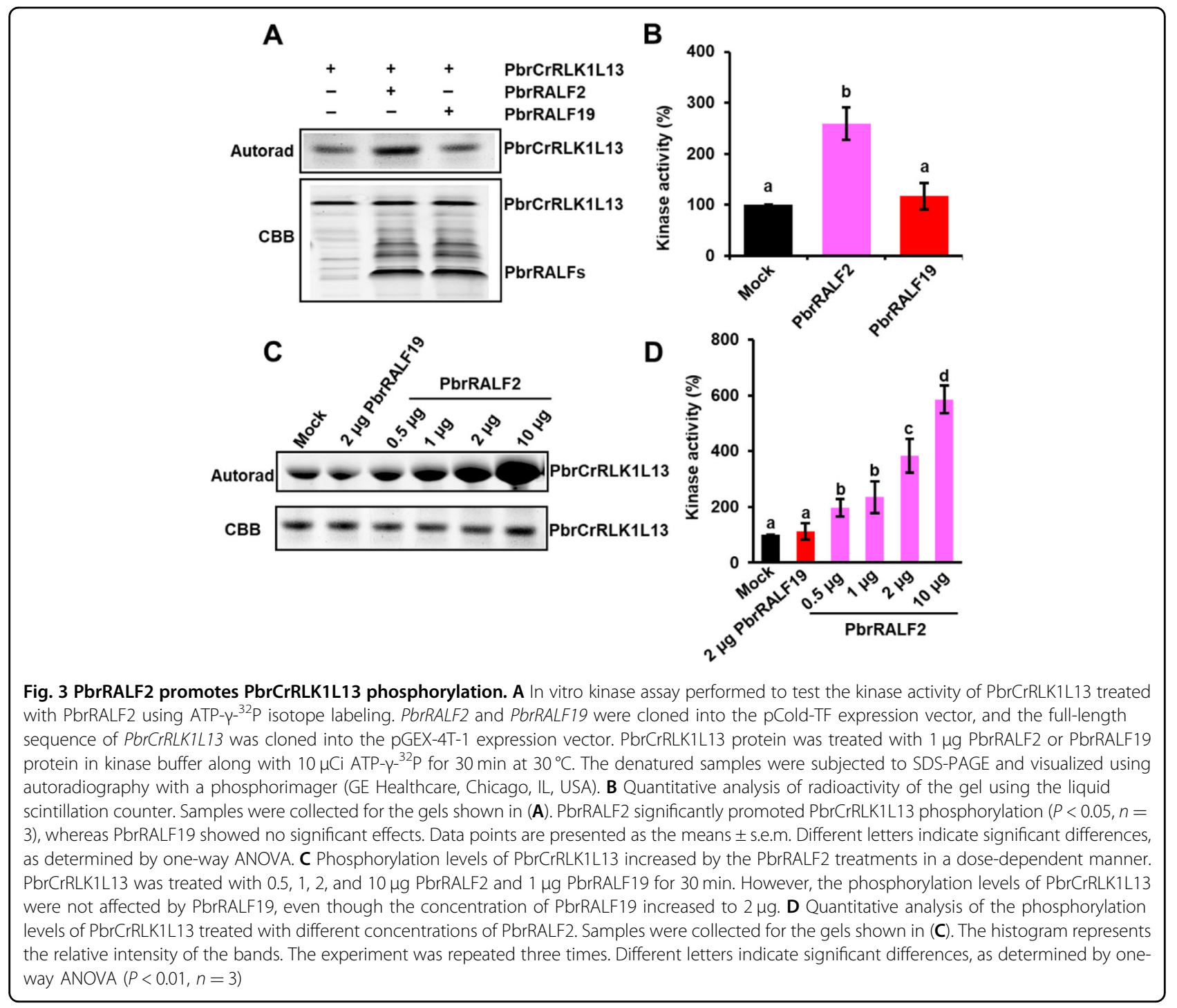

the production of ROS in Arabidopsis ${ }^{10}$, ROS production was significantly increased upon PbrRALF2 treatment in pear pollen (Fig. 4C and Supplementary Fig. S7A). Consistent with the lack of phosphorylation of PbrCrRLK1L13 in the presence of PbrRALF19, ROS production was also insensitive to PbrRALF19. Notably, when the expression of PbrCrRLK1L13 was knocked down, ROS production became insensitive to PbrRALF2 (Fig. 4D and Supplementary Fig. S7B). Together, these results suggest that PbrCrRLK1L13 mediates the PbrRALF2-elicited increase in ROS production and pollen tube growth inhibition in pear.

\section{PbrMPK18 is one of the downstream targets of PbrCrRLK1L13}

To identify intracellular downstream signaling molecules of PbrCrRLK1L13 in pollen tubes, we used the
PbrCrRLK1L13 kinase domain as bait to screen a cDNA library of pear pollen tubes by Y2H. PbrMPK18 was identified as an interactor of PbrCrRLK1L13 (Supplementary Table S3). The $\mathrm{Y} 2 \mathrm{H}$ assay (Fig. 5A), luciferase complementation imaging (LCI) (Fig. 5B), and bimolecular fluorescence complementation (BiFC) assays (Fig. 5C) revealed that PbrMPK18 interacts directly with PbrCrRLK1L13. The interaction level of PbrCrRLK1L13 and PbrMPK18 was significantly enhanced in the presence of PbrRALF2 (Fig. 6A). Conversely, when PbrMPK18 was knocked down using the antisense oligonucleotide approach, both ROS production (Fig. 6B and Supplementary Fig. S7C) and pollen tube growth (Fig. 6C) became insensitive to PbrRALF2. Thus, PbrMPK18 acts as a downstream component of PbrCrRLK1L13 to mediate ROS production and pollen tube growth inhibition in PbrRALF2 signaling (Fig. 6D). 


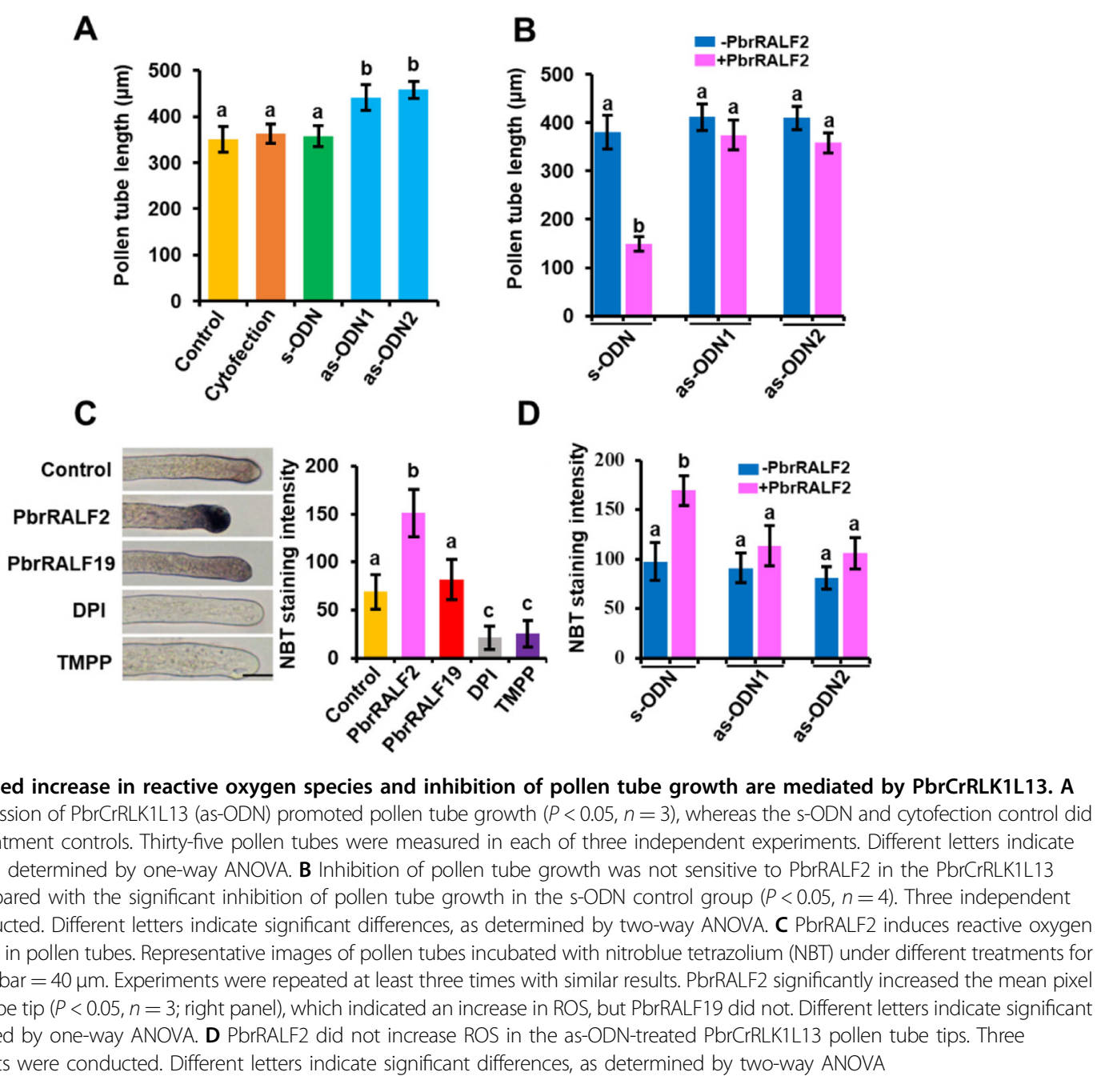

\section{Discussion}

\section{PbrRALF2 acts as an inhibitor to control the balance of} pollen tube growth

RALFs have long been identified as ubiquitous peptides in plants. Inhibitory effects of RALFs on roots s,32 $^{5,}$ and pollen tube growth have also been observed ${ }^{3,4,6}$. In this study, we identified a signaling pathway for PbrRALF2 that inhibits the growth of pear pollen tubes. Our results support a model in which PbrRALF2 is secreted into the apoplast during pollen tube elongation and then binds to PbrCrRLK1L13 and triggers its intracellular phosphorylation. This process leads to the inhibition of pollen tube elongation and thus controls the steady-state growth of the pollen tube.

Several lines of evidence suggest an inhibitory effect of PbrRALF2 on pollen tube growth. Both synthetic and purified E. coli-expressed PbrRALF2 induced dosedependent inhibition of pollen tube elongation (Fig. 1B). When PbrRALF2 was knocked down, the pollen tubes grew faster (Fig. 1C). The finding that PbrRALF2 inhibits pollen tube growth was initially puzzling because this peptide from pollen was produced by itself. However, it is possible that this growth inhibition is a form of autoregulation that allows the pollen tube to grow normally. Other pollen self-generated peptides are known to contribute to this type of regulation ${ }^{2}$, and accurate control of the rate of pollen tube growth is essential for successful fertilization.

\section{RALF signal was transduced into the cytosol via PbrCrRLK1L13}

Many peptides have been identified as apoplast signals, which exert their function using membrane receptors. RLKs are one such type of receptor in plants involved in the perception of extracellular signals and cytoplasmic interactor transduction ${ }^{33}$. For example, CLAVATA1, the receptor of CLV3, controls the size of the organizing center in the shoot apical meristem and regulates the 

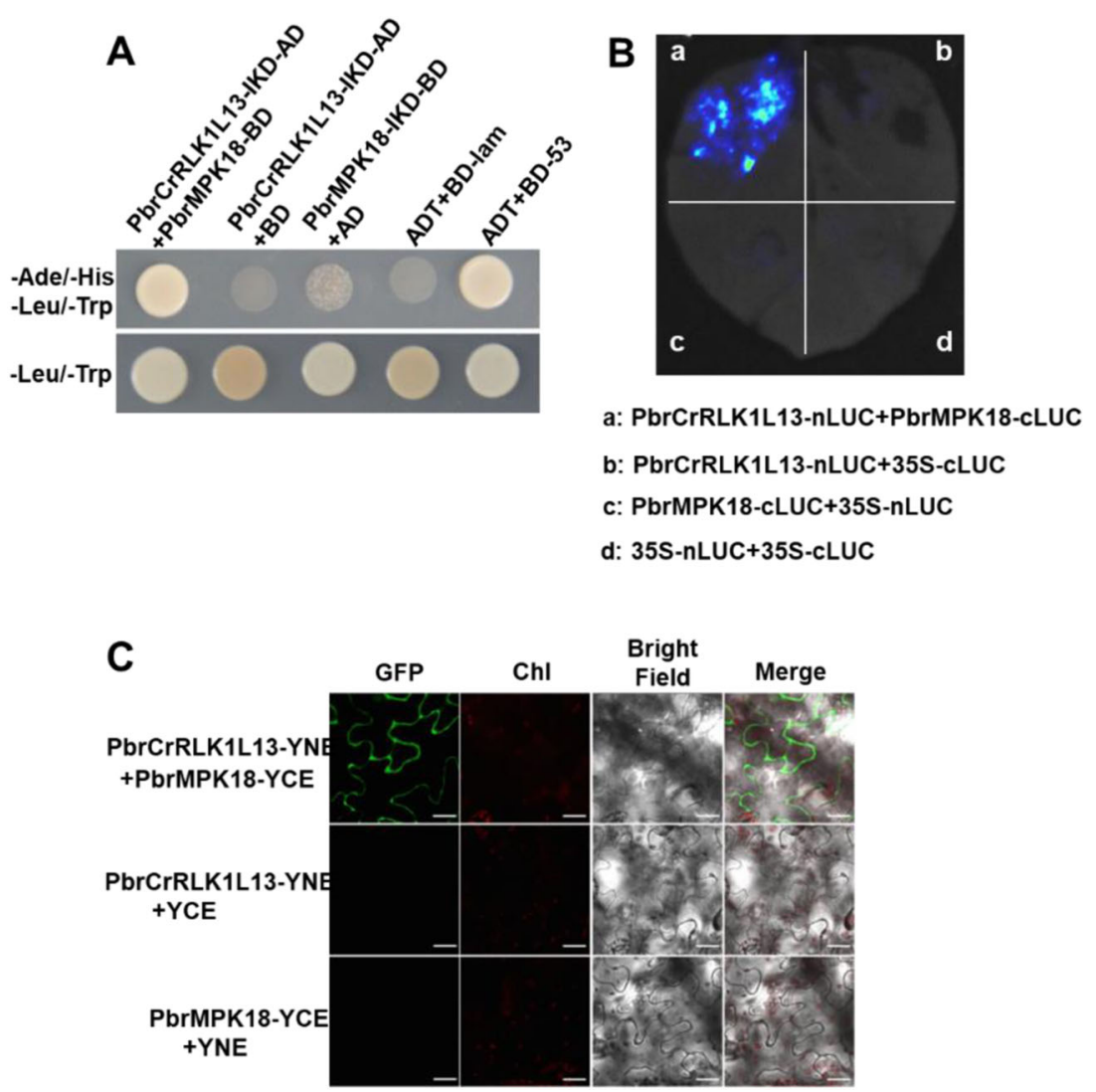

Fig. 5 PbrMPK18 interacts with PbrCrRLK1L13. A PbrMPK18 interacts with the intracellular domain of PbrCrRLK1L13 in yeast two-hybrid assays. The intracellular domain of PbrCrRLK1L13 fused to the pGADT7 vector and that of PbrMPK18 fused to the PGBKT7 vector. Growth in the medium that lacked Trp, Leu, His, and Ade indicated protein-protein interactions. B PbrMPK18 and PbrCrRLK1L13 interact in the plasma membrane of N. benthamiana leaf epidermal cells in the LCl assay. Fluorescence on the leaf surface indicated protein-protein interactions. C PbrMPK18 and PbrCrRLK1L13 interact in the plasma membrane of $\mathrm{N}$. benthamiana leaf epidermal cells in the BiFC assay. The GFP signal indicates the interaction between PbrMPK18 and PbrCrRLK1L13. Chl represents chloroplast autofluorescence. Merge represents the merged image of GFP, Chl, and bright field. Scale bars $=5 \mu \mathrm{m}$

expansion of plant roots ${ }^{34,35}$; and PSKR, as the receptor of PSK, regulates cell expansion during plant development ${ }^{36}$. In addition, pollen-specific RLK proteins regulate pollen tube growth. The receptor kinase LePRK2 transduces LAT52 information to control pollen hydration and germination $^{37}$. A pollen-specific receptor kinase in Arabidopsis is essential for pollen tube reception of the signal from the AtLURE1 peptide ${ }^{38,39}$. FER is a receptor for AtRALF1 to inhibit cell elongation in $\operatorname{roots}^{8,9}$ and AtRALF23 to inhibit plant immunity ${ }^{10,40}$. Here, we provide evidence that PbrCrRLK1L13 is a receptor for PbrRALF2 in pear pollen. This result represents another ligand-receptor pair involving a RALF and a CrRLK1L protein and highlights the diverse functions of this complex in regulating plant growth and cell-cell communication. PbrRALF2 can bind to PbrCrRLK1L13 and has submicromolar affinity (Kd) for PbrCrRLK1L13 (Fig. 2), thus meeting the criterion of ligand-receptor pairs ${ }^{13,41}$, and this affinity is consistent with the physiological concentration required for its inhibition of pollen tube growth (Fig. 1B).

Notably, PbrCrRLK1L13 belongs to the same clade as BUPS1 and BUPS2 of Arabidopsis and RUPO of rice $^{15,16,29}$. Based on our evidence and previous reports, we know that PbrCrRLK1L13, BUPS1, and BUPS2 are receptors for RALF peptides ${ }^{6}$ (Fig. 2). RUPO is known to facilitate male transmission by controlling the potassium concentration in pollen tube ${ }^{16}$, but further investigation is required to determine whether RUPO is also a receptor of RALF in the pollen tube of rice.

PbrCrRLK1L13 interacts with its PbrRALF2 ligand via exJM at the plasma membrane (Fig. 2B), similar to the region where LORELEI and LLG1 physically interact with $\mathrm{FER}^{42}$, thus implying a potentially conserved role for the exJM region of CrRLK1Ls. The phosphorylation of PbrCrRLK1L13 can be directly promoted by PbrRALF2 (Fig. 3), and knockdown of PbrCrRLK1L13 renders the pollen tube less sensitive to PbrRALF2 (Fig. 4B), 

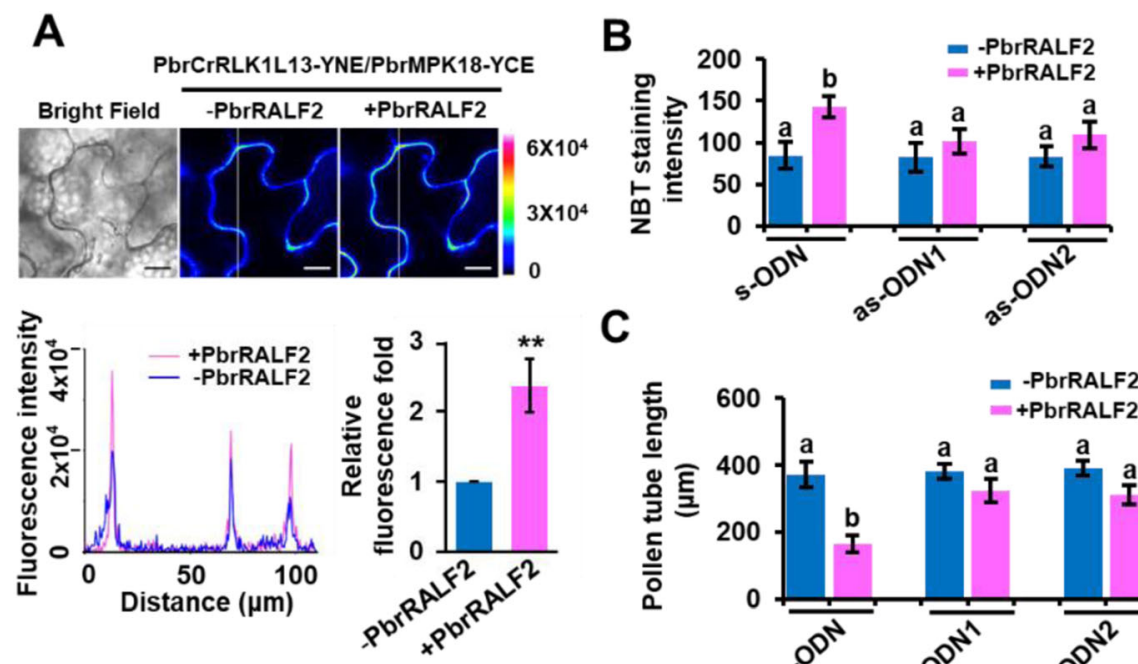

C
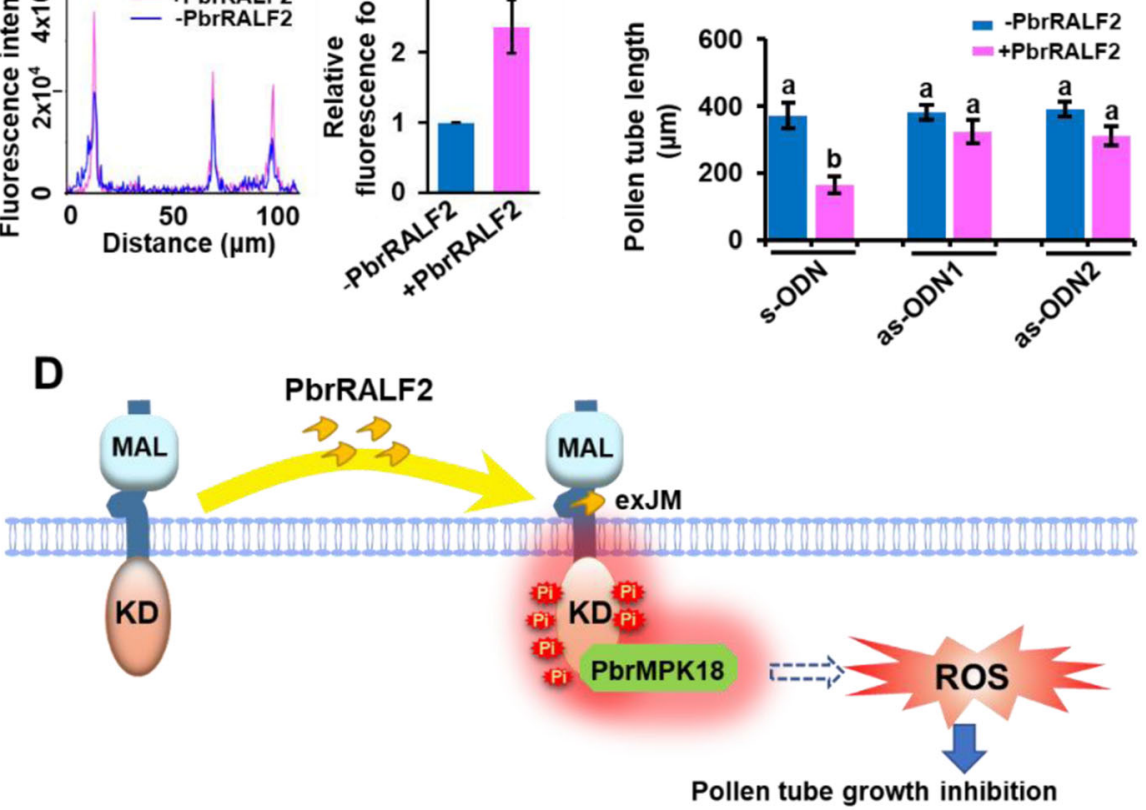

Fig. 6 PbrRALF2 enhances the PbrCrRLK1L13-PbrMPK18 interaction, and PbrMPK18 is required for PbrRALF2 signaling. A Interaction of PbrCrRLK1L13 with PbrMPK18 analyzed by BiFC in N. benthamiana epidermal cells. The interaction levels of PbrCrRLK1L13 and PbrMPK18 were significantly enhanced by $1 \mu$ M PbrRALF2. ${ }^{* *}$ represents $P<0.01$; Student's $t$ test). Data points are presented as the means \pm s.e.m. Scale bar $=20 \mu \mathrm{m}$. B PbrMPK18 is required for PbrRALF2-induced ROS accumulation. PbrMPK18 was knocked down by as-ODN1 and as-ODN2, with s-ODN as a negative control. PbrRALF2 $(1 \mu \mathrm{M})$ was used to treat the pollen tubes. ROS accumulation was tested by NBT staining. Thirty-five pollen tubes were measured in each of three independent experiments. Different letters indicate significant differences, as determined by two-way ANOVA. C Inhibition of pollen tube growth was not sensitive to PbrRALF2 when PbrMPK18 was knocked down by as-ODN in pollen tubes. Thirty-five pollen tubes were measured in each of three independent experiments. Different letters indicate significant differences, as determined by two-way ANOVA. D Proposed model for PbrRALF2 perception in pollen tubes of pear. After PbrRALF2 binds to the exJM region of PbrCrRLK1L13, phosphorylation of PbrCrRLK1L13 is enhanced. Under the mediation of PbrMPK18, phosphorylated PbrCrRLK1L13 increases ROS production in pollen tubes. Excessive ROS production eventually inhibits the growth of pollen tubes

indicating that the interaction of PbrRALF2 with PbrCrRLK1L13 is functionally relevant. Such phosphorylation by peptides has similarly been observed for multiple receptor-like kinase proteins. In Arabidopsis, RALF1 triggers $\mathrm{FER}^{8}$ and $\mathrm{BAK} 1^{41}$ phosphorylation to suppress root elongation. In the self-incompatibility response of Brassica, the pollen $S$-determinant $S$ locus cysteine-rich protein binds to its corresponding pistil $S$-determinant $S$ locus receptor kinase (SRK), resulting in the phosphorylation of SRK and initiation of a signal transduction cascade that ultimately results in pollen death ${ }^{43-46}$. Considering these results together, it seems possible that phosphorylation of the cytoplasmic kinase domains of membrane RLKs represents a general method of conveying extracellular peptide messages to cytosolic proteins.

\section{Enhanced PbrCrRLK1L13-induced ROS production is mediated by PbrMPK18}

MAP kinases can be activated by RALFs ${ }^{5}$, but it is not clear how RALFs transmit signals to MAPKs and which components encode RALF-responsive MAPKs. In addition to MKKs, MAPKs are also regulated by other kina$\operatorname{ses}^{47-49}$. Here, interaction studies revealed the binding of PbrCrRLK1L13 to PbrMPK18 (Fig. 5), indicating that PbrMPK18 should be considered a downstream target of PbrCrRLK1L13. Importantly, the interaction of PbrCrR LK1L13 with PbrMPK18 was enhanced by the PbrRALF2 
treatment (Fig. 6A). When PbrMPK18 was knocked down, pollen tube growth was insensitive to PbrRALF2 (Fig. 6C), thus providing more direct evidence that PbrMPK18 plays a key role in PbrRALF2 signaling. PbrMPK18 is closely related to Arabidopsis MPK6; thus, further work will be interesting to investigate whether MPK6 is involved in RALF signaling in Arabidopsis.

A central element of RLK signaling is the ROS burst $^{23,50}$. In FER-mediated signaling, RALF17 causes an increase in ROS production; however, RALF peptides have a predicted S1P cleavage site that leads to an inhibition of ROS production, and the specific mechanisms underlying the differences among RALFs remain unclear $^{10}$. ROS production is essential for cell signaling and regulation, although too much ROS can be harmful to cell homeostasis. At low levels, ROS can alter the intracellular redox state, activate redox-sensitive proteins, and modify redox-sensitive domains of proteins by inhibiting or boosting their enzymatic activity ${ }^{51,52}$, while at high levels, ROS damage DNA, proteins, phospholipids, and other macromolecules, thereby impairing physiological function ${ }^{53}$. Two classes of signaling components have been suggested as the main mediators of RLK stimulation of extracellular ROS production ${ }^{23}$. One is receptor-like cytoplasmic protein kinases, such as BIK, which mediate FLS2 and BIR1 signaling to produce ROS. The other includes small guanine nucleotide-binding proteins, such as GEF1, which mediate FER and AtPRK2 signaling to produce ROS. We observed here that activation of PbrCrRLK1L13 led to the production of ROS in a manner mediated by PbrMPK18 (Fig. 6B). These findings point to another mechanism for RLK-induced ROS production. However, the connection between MAPKs and ROS signaling remains elusive, and future work is necessary to unravel the underlying mechanisms.

Based on our results, we propose a model for a signaling event in which peptide-receptor interactions lead to the suppression of pollen tube growth (Fig. 6D). During pollen tube elongation, PbrRALF2 is predicted to bind to the exJM of receptor PbrCrRLK1L13, thereby promoting its intracellular phosphorylation. In a process mediated by PbrMPK18, the PbrRALF2-PbrCrRLK1L13 complex enhances ROS production, thereby leading to the suppression of pollen tube growth. Our results thus elucidate events during pollen tube growth and suggest that CrRLK1Ls generally serve as receptors for RALFs in plants.

\section{Materials and methods Plant materials}

Root, stem, leaf, petal, fruit, pollen, and pistil tissues from Pyrus bretschneideri Rehd. cv. Danshansuli pear trees were collected from the Fruit Experimental Yard of Nanjing Agricultural University. Pollen samples were preserved by air-drying at ambient temperature $\left(25^{\circ} \mathrm{C}\right)$ for
$12 \mathrm{~h}$ and subsequently stored at $-20^{\circ} \mathrm{C}$ with silica gel. Root, stem, leaf, petal, fruit, and pistil samples were frozen in liquid nitrogen and stored at $-80^{\circ} \mathrm{C}$ for RNA extraction.

Mesophyll protoplasts for protein subcellular localization analysis were extracted from Arabidopsis thaliana (Col-0) leaves grown under short-day $(8 \mathrm{~h}$ light and $16 \mathrm{~h}$ dark at $22^{\circ} \mathrm{C}$ ) conditions ${ }^{54}$. Nicotiana benthamiana seedlings were grown in the greenhouse with cycles of $16 \mathrm{~h}$ light and $8 \mathrm{~h}$ dark at $25^{\circ} \mathrm{C}$.

\section{Identification of RALF and CrRLK1L genes in pear}

To identify members of the $R A L F$ gene family in pear, the RALF domain sequence (PF05498) was used as a query for searching against the pear genome database (http:// peargenome.njau.edu.cn) with the HMMER3 software package. We identified $24 R A L F s$ in pear. All $R A L F$ genes with nonredundant hits and expected $E$ values $<0.01$ were collected. The predicted RALF proteins were confirmed using Pfam (http://pfam.sanger.ac.uk/search) and SMART (http://smart.embl-heidelberg.de/) tools.

\section{Expression and purification of the His-PbrRALF fusion protein}

The mature coding sequences for PbrRALF genes were amplified using PCR with BamHI and XbaI sites at the $5^{\prime}$ and $3^{\prime}$-ends; the primers for PbrRALFs are listed in Supplementary Table S4. The amplified PCR products were digested by BamHI and XbaI and ligated into the pColdTF DNA vector (Takara Bio, Dalian, China). The recombinant plasmids were individually transferred into E. coli BL21 (DE3) cells. Expression of the fusion protein (His-PbrRALFs) was induced by isopropylthio- $\beta$-D-1galactopyranoside (IPTG, $0.5 \mathrm{mM}$ ) after the culture reached an $\mathrm{OD}_{600}$ of 0.6 at $37^{\circ} \mathrm{C}$. The cultivation was continued at $15^{\circ} \mathrm{C}$ for $24 \mathrm{~h}$ with continuous shaking $(220 \mathrm{rpm})$, and the cells were subsequently collected by centrifugation for protein purification. The bacterial pellet from the $600 \mathrm{~mL}$ culture was resuspended in $30 \mathrm{~mL}$ lysis buffer $\left(50 \mathrm{mM} \mathrm{NaH}_{2} \mathrm{PO}_{4}, 300 \mathrm{mM} \mathrm{NaCl}, 10 \mathrm{mM}\right.$ imidazole, $\mathrm{pH}$ 7.9) and subsequently disrupted using an ultrasonic cell cracker (Model 705, Fisher Scientific, USA). The extract was centrifuged at $10,000 \times g$ for $15 \mathrm{~min}$ at $4{ }^{\circ} \mathrm{C}$, and the supernatant was recovered. The supernatant containing soluble proteins was added to a column containing $4 \mathrm{~mL}$ Ni-NTA His Bind Resin (EMD Millipore, MA, USA) and washed with $20 \mathrm{~mL}$ binding buffer $(20 \mathrm{mM}$ Tris- $\mathrm{HCl}$, $500 \mathrm{mM} \mathrm{NaCl}, 10 \mathrm{mM}$ imidazole, $\mathrm{pH} 7.9), 5 \mathrm{~mL}$ wash buffer $(20 \mathrm{mM}$ Tris- $\mathrm{HCl}, 500 \mathrm{mM} \mathrm{NaCl}, 100 \mathrm{mM}$ imidazole, $\mathrm{pH}$ 7.9), and $3 \mathrm{~mL}$ elution buffer $(20 \mathrm{mM}$ Tris- $\mathrm{HCl}$, $500 \mathrm{mM} \mathrm{NaCl}, 600 \mathrm{mM}$ imidazole, $\mathrm{pH} 7.9)$. The purified proteins were stored at $-80^{\circ} \mathrm{C}$. Protein quality was assessed by SDS/PAGE. 


\section{PbrRALF treatment assay}

First, the preservation solution of PbrRALFs was dialyzed (dialysis membrane, molecular cutoff 6,000-8,000) against $2 \mathrm{~L}$ of pollen culture medium $((\mathrm{w} / \mathrm{v}), 0.03 \% \mathrm{Ca}$ $\left(\mathrm{NO}_{3}\right)_{2} \cdot 4 \mathrm{H}_{2} \mathrm{O}, 10 \%$ sucrose, $0.01 \% \mathrm{H}_{3} \mathrm{BO}_{3}$, and $30 \mathrm{mM}$ MES, pH 6.3) at $4{ }^{\circ} \mathrm{C}$ for $16 \mathrm{~h}$. Subsequently, pear pollen samples were precultured in the basal medium at $25^{\circ} \mathrm{C}$ in darkness for $40 \mathrm{~min}$. Subsequently, a portion of the precultured pollen tubes was treated with PbrRALFs for $2 \mathrm{~h}$, and equivalent His-TF expressed from empty pCold-TF DNA vectors were used as the controls. The pollen tubes were photographed using an Olympus IX73 microscope (Olympus Optical, Japan, https://www.olympuslifescience.com). The length of the pollen tubes was measured with ImageJ software (https://imagej.nih.gov/ij).

\section{Quantitative RT-PCR}

The MIQE guidelines were followed for quantitative RT-PCR (qRT-PCR) experiments. The Plant Total RNA Isolation Kit (FOREGENE, Chengdu, China) was used to extract total RNA, and DNase I was used to eliminate genomic DNA contaminants. A RevertAid First Strand cDNA Synthesis Kit was used to reverse transcription purified total RNA ( $3 \mu \mathrm{g}$ ) (Thermo Scientific, USA). Then, using SYBR Green Master Mix on a LightCycler ${ }^{\circledR} 480$ II (Roche, Switzerland), quantitative PCR (qPCR) was performed on 20- $\mu \mathrm{l}$ samples according to the manufacturer's instructions. The thermal cycle was performed at $95^{\circ} \mathrm{C}$ for $5 \mathrm{~min}$, followed by 45 cycles of $15 \mathrm{~s}$ at $95^{\circ} \mathrm{C}$ and $15 \mathrm{~s}$ at $60^{\circ} \mathrm{C}$. The pear TUBULIN gene was used as an internal control, and relative expression levels were evaluated using the $2 \mathrm{CT} 2-\triangle \triangle \mathrm{CT}$ method, as previously described $^{55}$. The primers for RT-PCR and qRT-PCR of PbrRALFs, PbrCrRLK1L13, PbrMPK18, and PbrTUB in pear are presented in Supplementary Tables S5 and S6, respectively.

\section{ROS detection assays in pollen tubes}

To detect ROS production in the tip of the pollen tube after PbrRALF2 treatment, we used PbrRALF2 to treat pear pollen for $2 \mathrm{~h}$ at $25^{\circ} \mathrm{C}$. PbrRALF19 was used as a negative control. Pollen tubes were stained with nitroblue tetrazolium (NBT; $1 \mathrm{mg} \mathrm{mL}^{-1}$ ) for $5 \mathrm{~min}^{56}$. The stained samples were photographed using an Olympus IX73 microscope. ImageJ software was used to calculate the pixel intensity of formazan precipitation in the pollen tube tips. The pixel intensity values were calculated as previously described ${ }^{57}$. More than 35 pollen tubes were measured in each treatment, with three biological replicates. In addition, we further used $2^{\prime}, 7^{\prime}$-dichlorodihydrofluorescein (CM-H2DCFDA, final concentration $5 \mu \mathrm{M})$ to detect ROS production. The pollen tubes were then imaged by confocal microscopy on a Zeiss 880 LSCM microscope.

\section{Subcellular localization analysis}

The full-length coding sequence of pear PbrCrRLK1L13 was amplified using PCR from pear pollen CDNA with Phusion High-Fidelity DNA Polymerase (Thermo Scientific, USA). The following primers were synthesized with restriction sites: PbrCrRLK1L13 forward, 5'-GCTCTAGAATGGC TCTCCTCCTGGTCCT-3', and PbrCrRLK1L13 reverse, 5'GAAGATCTCCTACCATTTAGATTGGAAAATTG-3'.

The PCR products for PbrCrRLK1L13 were subsequently digested using XbaI and BglII. The digested PCR products were fused in frame with the sequence encoding GFP into $\mathrm{XbaI}$ and BamHI sites of the p35S:CDS-GFP vector ${ }^{58}$ to produce the plasmid $p 35 S: P b r C r R L K 1 L 13-G F P$, which was then transfected into Agrobacterium (GV3101). Agrobacterium-mediated infiltration in Nicotiana benthamiana leaves was performed with a needleless syringe as reported ${ }^{59}$. The images were acquired using a Zeiss LSM 780 (Germany, https://www.zeiss.com/microscopy) confocal laser-scanning microscope after 3 days of tobacco cultivation. All transient expression assays were repeated at least three times.

\section{Yeast two-hybrid assay}

PbrRALF2 was cloned into a pGBKT7 vector and used as bait in the AH109 strain to screen the pear pollen cDNA library. Yeast two-hybrid analyses were conducted as previously described ${ }^{60}$, and PbrCrRLK1L13 was identified as a candidate protein. To verify the interaction between PbrRALF2 and PbrCrRLK1L13, PbrRALF2 was cloned into the pGADT7 vector, and the sequence encoding the PbrCrRLK1L13 extracellular domain (aa 1-417) was cloned into the pGBKT7 vector. The constructed vectors were cotransformed into yeast, selected on SD/-Leu-Trp and verified on SD/-Leu-Trp-His-Ade. Then, the PbrCrRLK1L13 kinase domain (aa 441-770) was used as bait to screen the pear pollen cDNA library using the AH109 system. The method of screening and validation in yeast was similar to the interaction between PbrRALF2 and PbrCrRLK1L13. The primers used for the yeast two-hybrid assay are listed in Supplementary Table S7.

\section{Bimolecular fluorescence complementation}

For the $\mathrm{BiFC}^{61}$ assay, the coding sequence of PbrMPK18 was cloned into pSPYCE-35S, and the full-length sequence of PbrCrRLK1L13 was cloned into pSPYNE35S. Agrobacterium carrying the plasmid were coinfiltrated in $N$. benthamiana leaves. Confocal microscopy (Zeiss LSM 780, Germany, https://www.zeiss.com/ microscopy) images were captured 3 days after infiltration. The primers used for gene cloning and vector construction are listed in Supplementary Table S8.

\section{Luciferase complementation imaging assay}

A luciferase complementation imaging (LCI) assay was conducted. The full-length coding sequence of 
PbrCrRLK1L13 was cloned into the vector nLUC, and the full-length coding sequence of PbrMPK18 was cloned into the vector cLUC. Paired constructs of PbrMPK18-cLUC and PbrCrRLK1L13-nLUC were transiently coexpressed in the leaves of N. benthamiana through Agrobacteriummediated coinfiltration. The primers used for this assay are listed in Supplementary Table S9.

\section{Expression and purification of GST-PbrCrRLK1L13-ECD}

The region encoding the extracellular domain of PbrCrRLK1L13 was amplified by PCR and cloned into the pGEX-4T-1 vector. The corresponding primers with BamHI and Xhol sites were as follows: forward, 5'-CGGGAT CCATGGCGTCCTCCCCACC-3', and reverse, 5'-CCG CTCGAGCTTGTGAGGCCCGCCG-3'. The expression vectors were transformed into E. coli BL21 (DE3) and subcultured in $600 \mathrm{~mL}$ of Luria-Bertani (LB) medium until the $\mathrm{OD}_{600}$ reached 0.6. Then, the expression of GST (from the empty vector) or GST-PbrCrRLK1L13-ED was induced by IPTG $(0.5 \mathrm{mM})$, and the cells were further cultivated for $8 \mathrm{~h}$ at $26^{\circ} \mathrm{C}$. One milliliter of binding buffer $(25 \mathrm{mM}$ Tris $\bullet \mathrm{HCl}$, $\mathrm{pH} 7.5,100 \mathrm{mM} \mathrm{NaCl}$, and $1 \mathrm{mM}$ DTT) was used to prewash GST agarose resin (Thermo Fisher Scientific, USA) three times.

\section{In vitro phosphorylation assays}

The full-length sequence of $\operatorname{PbrCrRLK1L13}$ was cloned into the pGEX-4T-1 expression vector to produce GSTtagged recombinant protein in E. coli. Primers for PbrCrRLK1L13 were as follows: forward, 5'-GAAGATCT ATGGCTCTCCTCCTGGTCC-3', and reverse, 5'-CCGCTCGAGTTACCTACCATTTAGATTGGAAAA T-3'. The membrane proteins were expressed using MembraneMax ${ }^{\mathrm{TM}}$ Protein Expression Kits (Thermo Fisher Scientific, USA). Ni-NTA His Bind resin (EMD Millipore, MA, USA) was used to purify the protein, and then SDS/PAGE was performed to determine the protein purity. The reaction buffer was $\left[25 \mathrm{mM}\right.$ Tris $\bullet \mathrm{HCl}$ (pH 7.5), $1 \mathrm{mM} \mathrm{MnSO}_{4}$, $0.5 \mathrm{mM} \mathrm{CaCl} 2,2 \mathrm{mM}$ DTT, $10 \mu \mathrm{M}$ ATP, and $10 \mu \mathrm{Ci}$ of [y32] ATP (3000 Ci mmol-1)]. Kinase activity assays were conducted for $30 \mathrm{~min}$ at $30^{\circ} \mathrm{C}$. SDS-PAGE was used to analyze the denatured samples, which were then stained with Coomassie brilliant blue and autoradiographed with a phosphorimager (GE Healthcare, USA).

\section{Surface plasmon resonance assay}

Surface plasmon resonance (SPR) experiments were conducted at $25^{\circ} \mathrm{C}$ using a Biacore T200 (GE Healthcare, USA, https://www.biacore.com). On a CM5 sensor chip, PbrCrRLK1L13 immobilization was accomplished using the standard amine coupling technique with a running buffer of $10 \mathrm{mM}$ HEPES, $150 \mathrm{mM} \mathrm{NaCl}$, and $3 \mathrm{mM}$ EDTA at $\mathrm{pH}$ 7.4. With a contact time of $60-100 \mathrm{~s}$, a flow rate of $100 \mathrm{~L} / \mathrm{min}$, and a dissociation time of $200-300 \mathrm{~s}$, PbrRALF2 was injected onto the surface. By subtracting the signals on the reference surface from those on the target protein surface and the signals of the blank sample (no mimetics) from those of the mimetic-containing samples, the sensorgrams were double-corrected for nonspecific binding to the surface. With Biacore T200 Evaluation Software (version 2.0), the corrected signal was fitted to a steady-state 1:1 interaction model and the binding affinity (Kd) was obtained. GraphPad Prism was used to assess differences in Kd values for each test using one-way ANOVA and multiple Tukey's comparison tests (version 6.05).

\section{Isothermal titration calorimetry assay}

A MicroCal ITC200 instrument (Malvern Instruments, England, https://www.malvern.com) was used to acquire isothermal titration calorimetry (ITC) data at $25^{\circ} \mathrm{C}$. Using a PD-10 column, a $20 \mu \mathrm{M}$ PbrCrRLK1L13 solution was prepared in $50 \mathrm{mM}$ phosphate, $\mathrm{pH} 7.4$, and $150 \mathrm{mM} \mathrm{NaCl}$ (GE Healthcare, USA). PbrRALF2 solutions were produced as $200 \mu \mathrm{M}$ stocks in the same buffer. The sample cell was filled with $400 \mu \mathrm{L}$ of PbrCrRLK1L13 solution, and the syringe was filled with $40 \mu \mathrm{L}$ of PbrRALF2 solution in each ITC experiment. The PbrCrRLK1L13 solution was stirred at $1000 \mathrm{rpm}$. The titration began with a $0.2 \mu \mathrm{L}$ injection, followed by a series of 29 periodic injections of $1 \mu \mathrm{L}$ each. The data were analyzed using the evaluation software Origin 7 (MicroCal Inc., USA). Thermodynamic parameters were calculated using the "one set of sites" model and a nonlinear least-squares fit to the data.

\section{Gene knockdowns by antisense oligonucleotides (as-ODN)}

Twenty-mer phosphorothioate oligonucleotides against PbrRALF2, PbrCrRLK1L13, PbrMPK18, and their sense controls (s-ODN) were synthesized with three $5^{\prime}$ - and $3^{\prime}$ phosphorothioate-modified bases, and a scrambled mismatch sequence was used as a control. For the in vitro experiments, pear pollen grains were precultured at $25^{\circ} \mathrm{C}$ in darkness for $45 \mathrm{~min}$ in basal medium and pretreated with as-ODN and s-ODN (30 $\mu \mathrm{M}$ final concentration) with cytofectin $(15 \mu \mathrm{g} / \mathrm{ml})$ for $15 \mathrm{~min}$, with as-ODN and $\mathrm{s}$-ODN injected in the pollen medium. After $2 \mathrm{~h}$ of cultivation, the samples were photographed using an Olympus IX73 microscope. More than 35 pollen tubes were measured in each treatment, and the experiments were repeated at least three times. The ODN sequences are shown in Supplementary Table S10. For each gene, two as-ODNs were designed for the test. Two independent as-ODN1 and as-ODN2 with higher gene knockdown efficiency were chosen for further experiments (Supplementary Figs. S2B, S3B, and S6).

\section{Statistical analysis}

All experimental data represent at least three independent experiments and are presented as the mean \pm standard error 
(SE). GraphPad Prism (version 6.05) was used to analyze the data, and significant differences were compared using the Student's $t$ test for two groups of samples and ANOVA for multiple samples. Tukey's honestly significant difference test was used for multiple comparisons. ANOVA tables are provided in Supplementary Data S1.

\section{Acknowledgements}

This work was supported by the National Key Research and Development Program of China (2018YFD1000107), the National Natural Science Foundation of China (31772256 and 31772276), and the Priority Academic Program Development of Jiangsu Higher Education Institutions.

\section{Author contributions}

X.B.K. performed most of the experiments and wrote the manuscript. J.M.S., D. Q.W., and P.C. performed the protein expression assays. P.W. analyzed the data. J.L. and Y.H.C. provided the experimental materials. S.L.Z. revised the manuscript. J.Y.W. conceived the research and revised the manuscript. All authors read and approved the final manuscript.

\section{Data availability}

The authors confirm that the data in this study are available within the article and its supplementary materials. Original data can be requested from the corresponding authors.

\section{Conflict of interest}

The authors declare no competing interests.

Supplementary information The online version contains supplementary material available at https://doi.org/10.1038/s41438-021-00684-y.

Received: 7 April 2021 Revised: 26 July 2021 Accepted: 4 August 2021 Published online: 04 October 2021

\section{References}

1. Muschietti, J. et al. LAT52 protein is essential for tomato pollen development: pollen expressing antisense LAT52 RNA hydrates and germinates abnormally and cannot achieve fertilization. Plant J. 6, 321-338 (1994).

2. Chen, Y. F. et al. Peptide growth factor phytosulfokine-a contributes to the pollen population effect. Planta 211, 752-755 (2000).

3. Covey, P. A. et al. A pollen-specific RALF from tomato that regulates pollen tube elongation. Plant Physiol. 153, 703-715 (2010).

4. Mecchia, M. A. et al. RALF4/19 peptides interact with LRX proteins to control pollen tube growth in Arabidopsis. Science 358, 1600 (2017).

5. Pearce, G. et al. RALF, a 5-kDa ubiquitous polypeptide in plants, arrests root growth and development. Proc. Natl Acad. Sci. USA 98, 12843-12847 (2001).

6. Ge, Z. et al. Arabidopsis pollen tube integrity and sperm release are regulated by RALF-mediated signaling. Science 358, 1596 (2017).

7. Moussu, S. et al. Structural basis for recognition of RALF peptides by LRX proteins during pollen tube growth. Proc. Natl Acad. Sci. USA 117, 7494-7503 (2020).

8. Haruta, M. et al. Peptide hormone and its receptor protein kinase regulate plant cell expansion. Science 343, 408-411 (2014).

9. $\mathrm{Yu}, \mathrm{M}$. et al. The RALF1-FERONIA interaction modulates endocytosis to mediate control of root growth in Arabidopsis. Development 147, dev.189902 (2020).

10. Stegmann, M. et al. The receptor kinase FER is a RALF-regulated scaffold controlling plant immune signaling. Science 355, 287 (2017).

11. Duan, Q. et al. FERONIA controls pectin- and nitric oxide-mediated male-female interaction. Nature 579, 7800 (2020).

12. Escobar-Restrepo, J. M. et al. The FERONIA receptor-like kinase mediates male-female interactions during pollen tube reception. Science 317, 656-660 (2007).
13. Boisson-Dernier, A. et al. Disruption of the pollen-expressed FERONIA homologs ANXUR1 and ANXUR2 triggers pollen tube discharge. Development 136, 3279-3288 (2009)

14. Feng, $\mathrm{H}$. et al. LORELEI-LIKE GPI-ANCHORED PROTEINS 2/3 regulate pollen tube growth as chaperones and coreceptors for ANXUR/BUPS receptor kinases in Arabidopsis. Mol. Plant. 12, 1612-1623 (2019).

15. Lindner, H. et al. CrRLK1L receptor-like kinases: not just another brick in the wall. Curr. Opin. Plant. Biol. 15, 659-669 (2012).

16. Liu, L. et al. Receptor-like kinase RUPO interacts with potassium transporters to regulate pollen tube growth and integrity in Rice. PLoS Genet. 12, e1006085 (2016).

17. Miyazaki, S. et al. ANXUR1 and 2, sister genes to FERONIA/SIRENE, are male factors for coordinated fertilization. Curr. Biol. 19, 1327-1331 (2009).

18. Nissen, K. S. et al. Understanding CrRLK1L function: cell walls and growth control. Trends Plant Sci. 21, 516-527 (2016)

19. Hsi, L. C. et al. Opposing effects of 15-lipoxygenase-1 and -2 metabolites on MAPK signaling in prostate alteration in peroxisome proliferator-activated receptor y. J. Biol. Chem. 277, 40549-40556 (2002).

20. Pece, S. et al. Signaling from e-cadherins to the MAPK pathway by the recruitment and activation of epidermal growth factor receptors upon cell-cell contact formation. J. Biol. Chem. 275, 41227-41233 (2000).

21. Li, C. H. et al. The receptor-like kinase SIT1 mediates salt sensitivity by activating MAPK3/6 and regulating ethylene homeostasis in Rice. Plant Cell 26, 2538-2553 (2014)

22. Meng, X. et al. A MAPK cascade downstream of ERECTA receptor-like protein kinase regulates Arabidopsis inflorescence architecture by promoting localized cell proliferation. Plant Cell 24, 4948-4960 (2012).

23. Kimura, S. et al. Bound by fate: reactive oxygen species in receptor-like kinase signaling. Plant Cell 29, 638-654 (2017).

24. Boisson-Dernier, A. et al. ANXUR receptor-like kinases coordinate cell wall integrity with growth at the pollen tube tip via NADPH oxidases. PLoS Biol. 11, e1001719 (2013).

25. Chai, L. et al. MAP kinase PrMPK9-1 contributes to the self-incompatibility response. Plant Physiol. 174, 1226-1237 (2017).

26. De Graaf, B. et al. Self-incompatibility in Papaver targets soluble inorganic pyrophosphatases in pollen. Nature 444, 490-493 (2006).

27. Estruch, J. J. et al. Cloning and characterization of a maize pollen-specific calcium-dependent calmodulin-independent protein kinase. Proc. Nat. Acad. Sci. USA 91, 8837-8841 (1994).

28. Meng, D. et al. Decreased sorbitol synthesis leads to abnormal stamen development and reduced pollen tube growth via an MYB transcription factor, MdMYB39L, in apple (Malus domestica). New Phytol. 217, 641-656 (2018).

29. Kou, X. et al. Evolution, expression analysis, and functional verification of Catharanthus roseus RLK1-like kinase (CrRLK1L) family proteins in pear (Pyrus bretchneideri). Genomics 109, 290-301 (2017).

30. Li, C. et al. FERONIA and her pals: functions and mechanisms. Plant Physiol. 171, 2379-2392 (2016).

31. Wang, J. et al. Allosteric receptor activation by the plant peptide hormone phytosulfokine. Nature 525, 265-268 (2016).

32. $\mathrm{Wu}$, J. et al. NaRALF, a peptide signal essential for the regulation of root hair tip apoplastic $\mathrm{pH}$ in Nicotiana attenuata, is required for root hair development and plant growth in native soils. Plant J. 52, 877-890 (2010).

33. Muschietti, J. P. et al. How many receptor-like kinases are required to operate a pollen tube. Curr. Opin. Plant. Biol. 41, 73-82 (2018).

34. Brand, $U$. et al. Dependence of stem cell fate in Arabidopsis on a feedback loop regulated by CLV3 activity. Science 289, 617-619 (2000).

35. Galli, M. et al. Expanding the regulatory network for meristem size in plants Trends Genet. 32, 372-383 (2016).

36. Matsubayashi et al. An LRR receptor kinase involved in perception of a peptide plant hormone, Phytosulfokine. Science 296, 1470-1472 (2002).

37. Tang, W. A Cysteine-rich extracellular protein, LAT52, interacts with the extracellular domain of the pollen receptor kinase LePRK2. Plant Cell 14 2277-2287 (2002)

38. Takeuchi, H. et al. Tip-localized receptors control pollen tube growth and LURE sensing in Arabidopsis. Nature 531, 245-248 (2016).

39. Wang, T. et al. A receptor heteromer mediates the male perception of female attractants in plants. Nature 531, 241-244 (2016)

40. Xiao, Y. et al. Mechanisms of RALF peptide perception by a heterotypic receptor complex. Nature 572, 270-274 (2019). 
41. Dressano, K. et al. BAK1 is involved in AtRALF1-induced inhibition of root cell expansion. PLoS Genet. 13, e1007053 (2017).

42. Li, C. et al. Glycosylphosphatidylinositol-anchored proteins as chaperones and co-receptors for FERONIA receptor kinase signaling in Arabidopsis. elife $\mathbf{4}$ e06587 (2015).

43. Doucet, J. et al. Pollen acceptance or rejection: a tale of two pathways. Trends Plant Sci. 21, 1058-1067 (2016)

44. Nasrallah, J. B. Recognition and rejection of self in plant reproduction. Science 296, 305-308 (2002)

45. Nasrallah, J. B. Plant mating systems: self-incompatibility and evolutionary transitions to self-fertility in the mustard family. Curr. Opin. Genet Dev. 47, 54 (2017).

46. Schopfer, C. R. The male determinant of self-incompatibility in Brassica. Science 286, 1697-1700 (1999).

47. Ludwig, A. A. et al. Ethylene-mediated cross-talk between calcium-dependent protein kinase and MAPK signaling controls stress responses in plants. Proc. Nat. Acad. Sci. USA 102, 10736-10741 (2005).

48. Moon, $\mathrm{H}$. et al. NDP kinase 2 interacts with two oxidative stress-activated MAPKs to regulate cellular redox state and enhances multiple stress tolerance in transgenic plants. Proc. Nat. Acad. Sci. USA 100, 358-363 (2003).

49. Takahashi, F. et al. Calmodulin-dependent activation of MAP kinase for ROS homeostasis in Arabidopsis. Mol. Cell 41, 649-660 (2011).

50. Kimura, S. et al. CRK2 and c-terminal phosphorylation of NADPH oxidase $\mathrm{RBOHD}$ regulate reactive oxygen species production in Arabidopsis. Plant Cell 32, 1063-1080 (2020).
51. Forman, H. J. Use and abuse of exogenous $\mathrm{H} 2 \mathrm{O} 2$ in studies of signal transduction. Free Radic. Biol. Med. 42, 926-932 (2007).

52. Reth, M. Hydrogen peroxide as second messenger in lymphocyte activation. Nat. Immunol. 3, 1129-1134 (2002).

53. David, S. G. et al. Laboratory and clinical studies of cancer chemoprevention by antioxidants in berries. Carcinogenesis 29, 1665-1674 (2008).

54. Yoo, S. D. et al. Arabidopsis mesophyll protoplasts: a versatile cell system for transient gene expression analysis. Nat. Protoc. 2, 1565-1572 (2007).

55. Livak, K. J. et al. Analysis of relative gene expression data using real-time quantitative PCR. Methods 25, 402-408 (2002).

56. Wang, C. L. et al. S-RNase disrupts tip-localized reactive oxygen species and induces nuclear DNA degradation in incompatible pollen tubes of Pyrus pyrifolia. J. Cell Sci. 123, 4301-4309 (2011).

57. Potocký, M. et al. Reactive oxygen species produced by NADPH oxidase are involved in pollen tube growth. N. Phytol. 174, 742-751 (2007).

58. Xie, Q. et al. LNK1 and LNK2 are transcriptional coactivators in the Arabidopsis circadian oscillator. Plant Cell. 26, 2843-2857 (2014).

59. Sparkes, I. A., Runions, et al. Rapid, transient expression of fluorescent fusion proteins in tobacco plants and generation of stably transformed plants. Nat. Protoc. 1, 2019-2025 (2006).

60. Brückner, A. et al. Yeast two-hybrid, a powerful tool for systems biology. Int. J. Mol. Sci. 10, 2763-2788 (2009).

61. Walter, M. et al. Visualization of protein interactions in living plant cells using bimolecular fluorescence complementation. Plant J. 40, 428-438 (2010). 